\title{
Cost-Effectiveness of Second-Line Chemotherapy/ Biologics among Elderly Metastatic Colon Cancer Patients
}

Zhiyuan Zheng • Eberechukwu Onukwugha • Nader Hanna •

Kaloyan Bikov $\cdot$ Brian Seal $\cdot$ C. Daniel Mullins

To view enhanced content go to www.advancesintherapy.com

Received: April 22, 2014 / Published online: July 15, 2014

(C) The Author(s) 2014. This article is published with open access at Springerlink.com

\section{ABSTRACT}

Introduction: Advancements in chemotherapy treatment have improved the clinical management of metastatic colon cancer (mCC) patients. An increasing number of elderly mCC patients receive various combinations of regimens in second-line chemotherapy/biologics treatment (Tx2) after first-line treatment (Tx1) to prolong survival and/or palliate symptoms, but these regimens have higher costs. This analysis investigated the survival benefit and incremental cost associated with Tx2 among elderly mCC patients.

Electronic supplementary material The online version of this article (doi:10.1007/s12325-014-0134-8) contains supplementary material, which is available to authorized users.

Z. Zheng $(\varangle) \cdot$ E. Onukwugha · K. Bikov ·

C. D. Mullins

University of Maryland School of Pharmacy,

Baltimore, MD, USA

e-mail: jason.zheng@cancer.org

N. Hanna

Division of General and Oncologic Surgery,

Department of Surgery, University of Maryland

School of Medicine, Baltimore, MD, USA

B. Seal

Bayer Healthcare Pharmaceuticals, Inc.,

Wayne, NJ, USA
Methods: Elderly $\quad$ (aged $\geq 66$ years) SEERMedicare patients diagnosed with mCC in 2003-2007 were identified and followed until death or the end of 2009. Cox regression and partitioned least squares regression were utilized to obtain the survival benefit and incremental cost associated with Tx2 within a 5-year study period. A time-varying model was used to reduce bias due to sequential ordering of $\mathrm{Tx} 1$ and Tx2. The regressions controlled for patient demographic characteristics, clinical variables, and a proxy for poor performance. Bootstrapping was used to generate 95\% confidence intervals (CI).

Results: Of the 3,266 elderly mCC patients who received Tx1, 2,744 (84\%) died within the observation period; 1,440 (44\%) received Tx2. The survival benefit associated with receipt of Tx2 was 0.33 years (95\% CI 0.19-0.43), and the associated incremental cost was \$40,888 (95\% CI 3,044-44,324). The incremental costeffectiveness ratio (ICER) for Tx2 was $\$ 123,903$ per life year gained (95\% CI 9,600-216,082).

Conclusion: The estimated survival benefit of receiving second-line chemotherapy/biologics was about 4 months, which is consistent with evidence from clinical trials. This improved 
survival was associated with an ICER that exceeds the traditional threshold.

Keywords: Biologics; Chemotherapy; Costeffectiveness analysis; Inverse probability weighting Cox regression; Metastatic colon cancer; Oncology; SEER-Medicare; Timevarying modeling

\section{INTRODUCTION}

Chemotherapy drugs and biologics developed over the past decade have advanced the management of first-line treatment (Tx1) and second-line treatment (Tx2) for metastatic colon cancer (mCC) patients. New treatments come with improved survival and also with significant increases in the cost of care for mCC patients. The various combinations of regimens utilized in Tx1 and Tx2 in real-world practice impose challenges in understanding the incremental survival benefit as well as the associated cost with respect to the receipt of $\mathrm{Tx} 2$. A comprehensive examination of $\mathrm{Tx} 2$ as compared to $\mathrm{Tx} 1$ alone provides valuable information that can help clinicians and mCC patients make treatment decisions with respect to the benefits and costs of multiple lines of chemotherapy drugs and biologics. A detailed analysis of the incremental cost of Tx2 among elderly mCC patients can assist potential stakeholders to better understand the cost implications of improving the delivery of high-quality cancer care.

The National Comprehensive Cancer Network colon cancer guideline recommends a list of chemotherapy drugs [i.e., 5-fluorouracil (5-FU) and leucovorin (LV), irinotecan (IRI) and oxaliplatin (OX)] and biologics [i.e., bevacizumab (BEV), cetuximab, and panitumumab] for mCC patients. IRI and OX
(IROX) can also be administered together with/ without 5-FU/LV to mCC patients [1]. These primary treatments help to palliate symptoms and prolong survival. The recommended management strategies are based on evidence from randomized clinical trials (RCTs) [2-11]. However, real-world benefit of Tx2 among elderly mCC patients who have multiple comorbidities may differ from those enrolled in RCTs. Furthermore, a large portion of elderly mCC patients who receive Tx1 may not receive $\mathrm{Tx} 2$ or subsequent treatment line (TxS) due to multiple comorbid conditions, tumor responses, and severe side effects $[12,13]$. Retrospective cohort studies are useful to assess the effectiveness of $\mathrm{Tx} 2$ as compared to $\mathrm{Tx} 1$ for mCC, which provides complementary information to those obtained from RCTs to improve clinical practices to 'real world' patients, especially populations not studied in RCTs.

Given the rising cost of oncology care, the survival benefit and incremental cost associated with Tx2 for elderly mCC patients are particularly relevant to payers, such as Medicare. Moreover, the real-world survival benefit of $\mathrm{Tx} 2$, overall cost and costeffectiveness of Tx2 can also help clinicians to communicate with patients more efficiently and help patients make informed decisions regarding their treatment.

\section{METHODS}

\section{Data}

The surveillance epidemiology and end results (SEER)-Medicare data were used to identify elderly Medicare beneficiaries with mCC diagnoses. The SEER-Medicare data are the result of the linkage between SEER cancer registry data and Medicare claims data. The 
SEER program was conducted by the National Cancer Institute to provide demographic and clinical information for all incident cancer cases in 17 geographic regions in the USA. Medicare, administered by the Centers for Medicare and Medicaid Services, is the primary healthcare provider for the elderly (aged $\geq 65$ years) in the USA, which collects information about covered healthcare services from time of enrollment until death.

The analysis in this article is based on previously conducted studies, and does not involve any new studies of human or animal subjects performed by any of the authors. The approved Institutional Review Board protocol number is HP-00049426.

\section{Inclusion and Exclusion Criteria}

The study population consisted of SEERMedicare enrollees (aged $\geq 66$ years at the time of diagnosis) diagnosed with mCC between 2003 and 2007. Tumor location was identified using the site recode variable in SEER-Medicare, ranging from 15 to 23 $(15=$ cecum,$\quad 16=$ appendix,$\quad 17=$ ascending colon, $18=$ hepatic flexure, $19=$ transverse colon, $20=$ splenic flexure, $21=$ descending colon, $22=$ sigmoid colon, $23=$ large intestine). All patients needed to have Medicare Parts A and B for a full year prior to cancer diagnoses for accurate ascertainment of baseline comorbidities; therefore, patients enrolled in a managed care plan within 1 year prior to cancer diagnosis were excluded. Those who were diagnosed post-mortem were also excluded. The remaining patients were followed up through death or the end of 2009. Patients were censored from the study upon loss of complete Medicare coverage or enrollment in a managed care plan.

\section{Identification of Treatment Lines and Associated Costs}

The algorithm used for identifying treatment lines was developed by Bikov et al. [14], which is a claim-based algorithm used to identify initial and subsequent treatment lines. This study adopted the Medicare system perspective in the cost analysis and included direct medical costs reimbursed under Medicare Parts A and B, i.e., drug costs, hospitalization costs and physician fees.

\section{Patient-Level Factors Included in the Survival and Cost Analyses}

Patient-level clinical variables included poorly graded tumor, surgery of primary site, and surgery of liver metastases. Indicators were created for poorly graded tumor and surgery of primary site, respectively. A categorical variable was created for surgery of liver metastases: (1) none; (2) surgery to other sites; and (3) ablation of liver metastases with/out surgery to other sites. Charlson comorbidity index (CCI) was measured using claims within 1 year prior to cancer diagnosis [15, 16]. A proxy for poor performance status was created to detect any hospital bed use, oxygen use, walking aid use or wheel chair use within 3 months prior to cancer diagnosis [17]. Patient demographic information such as age, gender, marital status, urban living status, and race/ethnicity were also included in regression analyses. Age was defined as a categorical variable (each 10-year increment in age at the time of diagnosis). Race/ethnicity was categorized into Non-Hispanic White, African American, Hispanic White, and Asian. Contextual variables, i.e., indicators for year of diagnosis (2003-2007) and the SEER registry sites were also included in the analyses. 


\section{Statistical Methods}

Patient-level factors were examined by the number of chemotherapy/biologics treatment lines: no treatment (Tx); Tx1 only; or Tx2, which included those who received two or more lines. The column distributions of each patient-level factor for the Tx 1 only group and the Tx 2 group were compared with the no Tx group. In this unadjusted analysis, $p$ values were generated to measure the statistical differences between the corresponding column percentages, where the no Tx group was the reference group.

Because there is a sequential ordering from Tx1 to Tx2, on average patients who received $\mathrm{Tx} 2$ lived longer than those who received Tx1 only. A time-varying Cox regression framework was used to examine the incremental survival benefit associated with $\mathrm{Tx} 2$ to address this "immortal time bias" [18]. The time-varying modeling approach assigned a patient in the 'control' group during the time period of Tx1 and switched her/him to the 'experiment' group at the time of initiation of Tx2. As compared with the static modeling approach, where patients were categorized into two groups according to their final treatment status (received Tx1 only versus Tx2), the timevarying modeling approach took the dynamic process of patients' change of treatment status into consideration, which reduced the bias of the survival benefit associated with Tx2. We conducted both static and time-varying modeling approaches and compared the survival benefits associated with Tx 2 using the Cox regression framework.

Individual mCC patients had different probabilities of receiving treatment(s) according to a specific set of patient-level clinical and demographic variables. The inverse probability weighting (IPW) method was used to control for the self-selection issue into treatment(s).
Multivariate sequential logistic regressions were utilized to estimate individual patients' probabilities of receiving Tx1, Tx2 and TxS, respectively. Two more variables were added in this step: (1) state buy-in status, which is indicative of low socioeconomic status; and (2) household median income, which measures the income level of the neighborhood defined by the zip code of the patient's residence. We used this variable as an additional proxy for patients' socioeconomic status, as has been done in prior studies [19]. The combination of the timevarying Cox regression framework and IPW method offered a flexible framework, where individual patients' IPW weights were updated depending on their treatment status, e.g., switching from Tx1 to Tx2.

Patients were also subject to censoring over time. Each month, a certain percentage of identified mCC patients were censored either due to end of study or switching to a health maintenance organization plan. The Lin's regression method was proposed to reduce the bias due to patients' missing information of their future cost accumulation elsewhere [20]. This method inflates the costs of those patients who are still alive and uncensored proportionally to the percentage of patients who are censored during each month. The monthly incremental cost associated with Tx2 was obtained by a multivariate regression, which measures the difference in costs of patients who were actively in Tx2 with those who were actively in Tx1. The overall incremental cost of Tx2 over the 5-year period was the sum of all 60 monthly costs that could be attributed to Tx2 versus Tx1 [21]. An elderly mCC patient would be in the 'control' group (Tx1 group) and switched to the comparison group (i.e., Tx2 group) later on according to the treatment status in a particular month. Therefore, the cost analysis of Tx 2 is also time 
varying in essence. The incremental costeffectiveness ratio (ICER) was created by the ratio of overall incremental cost and survival benefits associated with $\mathrm{Tx} 2$.

The static modeling of the incremental cost associated with Tx2 was obtained by comparing the Tx2 group with the Tx1 only group according to their final treatment status. Similar multivariate regressions were conducted as in the time-varying approach; however, the control group and comparison group remained unchanged over time. The corresponding ICER of the static modeling was also generated. Both time-varying and static modeling approaches used bootstrapping method to generate the $95 \%$ confidence intervals (CI) for the ICERs [22]. We assumed that there is a positive rate of time preference that patients would like to postpone cost in the future, thus separate analyses were conducted to discount the cost at 3 and 5\% annually to year 2010 dollars [23]. The statistical significance level was set a priori at $\alpha=0.05$.

\section{RESULTS}

As shown in Fig. 1, out of the 7,950 elderly mCC patients in the dataset, only 3,266 (41\%) received any treatment, and $44 \%$ of $\mathrm{Tx} 1$ users
$(1,440$ out of 3,266$)$ received Tx 2 . Receipt of Tx2 was associated with a lower 1-year mortality rate (13\%) compared with those who did not receive chemotherapy treatment $(88 \%)$ or received $\mathrm{Tx} 1$ only (53\%). The median time from diagnosis (Dx) to Tx1 and Tx2 was approximately 2 months and 12 months, respectively.

Table 1 shows the distribution of number of treatment lines by patient-level factors. In the unadjusted analysis, all clinical variables had an impact on the receipt of treatment: (1) higher CCI was associated with lower utilization rate of any treatment(s); (2) poorly graded tumor was associated with higher likelihood of receiving any chemotherapy treatment; (3) indicator for surgery of primary site increased the likelihood of receiving Tx1 and Tx2; and (4) patients who had ablation of liver metastasis or surgeries to other sites were associated with higher likelihood of receiving Tx1 and Tx2. Elderly mCC patients with the indicator of proxy for poor performance status had less chance of receiving chemotherapy treatment. Being older at the time of diagnosis was associated with lower likelihood of receiving Tx1, Tx2 and TxS. Being married was associated with higher percentages of patients receiving further treatment. Non-Hispanic White race was associated with higher utilization rate of

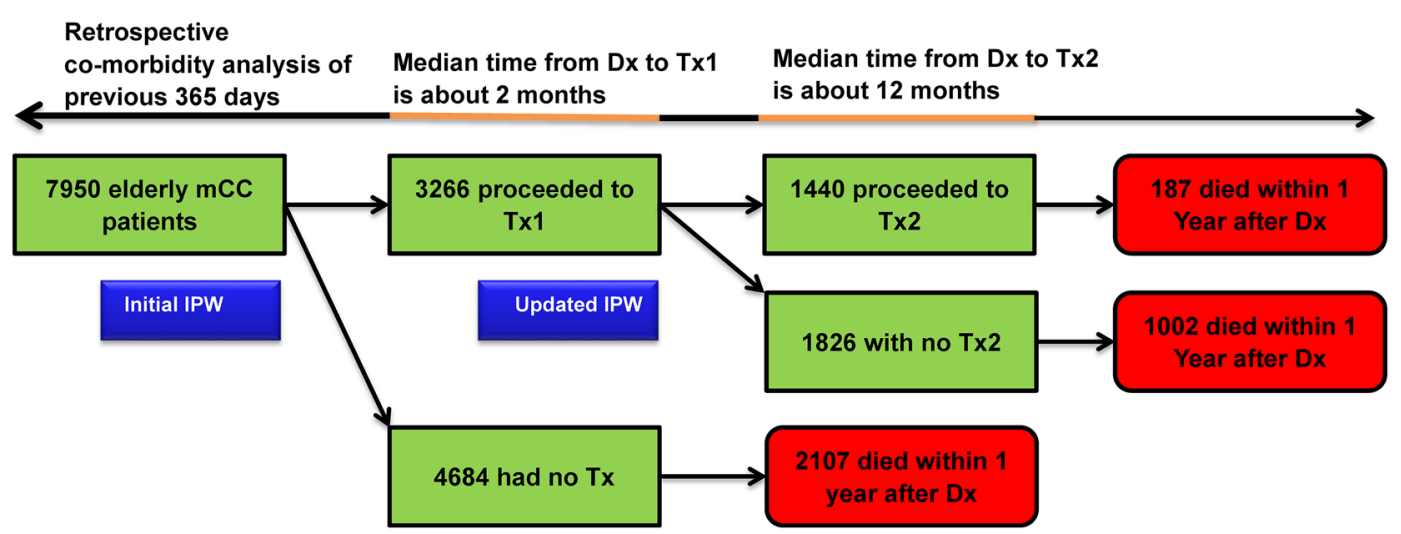

Fig. 1 A diagram of elderly mCC patients switching to Tx1 and Tx2. $D x$ diagnosis, $I P W$ inverse probability weighting, $m C C$ metastatic colon cancer, $T x 1$ first-line treatment, $T x 2$ second-line treatment 
Table 1 Distribution of number of treatment lines by patient-level factors

\begin{tabular}{|c|c|c|c|c|c|c|c|c|}
\hline \multirow[t]{3}{*}{ Total $(N=7,950)$} & \multirow{2}{*}{\multicolumn{2}{|c|}{$\frac{\text { No } T x}{(N=4,684)}$}} & \multirow{2}{*}{\multicolumn{3}{|c|}{$\frac{\text { Tx1 only }}{(N=1,826)}$}} & \multirow{2}{*}{\multicolumn{3}{|c|}{$\frac{T \times 2}{(N=1,440)}$}} \\
\hline & & & & & & & & \\
\hline & $\mathbf{N}$ & Col \% & $\mathbf{N}$ & Col \% & $p^{*}$ & $\mathbf{N}$ & Col \% & $p^{\dagger}$ \\
\hline \multicolumn{9}{|l|}{$\mathrm{CCI}$} \\
\hline $\mathrm{CCI}=0$ & 2,667 & 57 & 1,160 & 64 & \multirow[t]{4}{*}{$<0.0001$} & 975 & 68 & \multirow[t]{4}{*}{0.014} \\
\hline $\mathrm{CCI}=1$ & 1,023 & 22 & 424 & 23 & & 312 & 22 & \\
\hline $\mathrm{CCI}=2$ & 530 & 11 & 150 & 8 & & 97 & 7 & \\
\hline $\mathrm{CCI}=\geq 3$ & 464 & 10 & 92 & 5 & & 56 & 4 & \\
\hline \multicolumn{9}{|l|}{ Poorly graded tumor } \\
\hline Yes & 1,105 & 24 & 542 & 30 & \multirow{2}{*}{$<0.0001$} & 395 & 27 & \multirow[t]{2}{*}{0.033} \\
\hline No & 3,579 & 76 & 1,284 & 70 & & 1,045 & 73 & \\
\hline \multicolumn{9}{|l|}{ Surgery of primary site } \\
\hline Yes & 2,293 & 49 & 1,295 & 71 & \multirow[t]{2}{*}{$<0.0001$} & 1,139 & 79 & \multirow[t]{2}{*}{$<0.0001$} \\
\hline No & 2,391 & 51 & 531 & 29 & & 301 & 21 & \\
\hline \multicolumn{9}{|l|}{ Surgery of liver metastases } \\
\hline Ablation of liver metastasis & 319 & 7 & 257 & 14 & \multirow[t]{3}{*}{$<0.0001$} & 211 & 15 & \multirow[t]{3}{*}{0.515} \\
\hline Surgery to other site $(s)$ & 173 & 4 & 98 & 5 & & 64 & 4 & \\
\hline None & 4,192 & 90 & 1,471 & 81 & & 1,165 & 81 & \\
\hline \multicolumn{9}{|c|}{ Proxy for poor performance status } \\
\hline Yes & 601 & 13 & 104 & 6 & \multirow[t]{2}{*}{$<0.0001$} & 44 & 3 & \multirow[t]{2}{*}{$<0.0001$} \\
\hline No & 4,083 & 87 & 1,722 & 94 & & 1,396 & 97 & \\
\hline \multicolumn{9}{|l|}{ Age at time of $\mathrm{Dx}$} \\
\hline $66-74$ & 1,100 & 23 & 809 & 44 & \multirow[t]{3}{*}{$<0.0001$} & 797 & 55 & \multirow[t]{3}{*}{$<0.0001$} \\
\hline $75-84$ & 2,057 & 44 & 841 & 46 & & 592 & 41 & \\
\hline$\geq 85$ & 1,599 & 33 & 176 & 10 & & 51 & 4 & \\
\hline \multicolumn{9}{|l|}{ Female } \\
\hline Yes & 2,671 & 57 & 952 & 53 & \multirow[t]{2}{*}{$<0.0001$} & 708 & 49 & \multirow[t]{2}{*}{0.004} \\
\hline No & 2,013 & 43 & 874 & 47 & & 732 & 51 & \\
\hline Married & & & & & & & & \\
\hline Yes & 1,739 & 37 & 992 & 54 & $<0.0001$ & 905 & 63 & $<0.0001$ \\
\hline No & 2,945 & 63 & 834 & 46 & & 535 & 37 & \\
\hline Urban living area & & & & & & & & \\
\hline Yes & 4,197 & 90 & 1,620 & 89 & 0.068 & 1,309 & 91 & 0.021 \\
\hline No & 487 & 10 & 206 & 11 & & 131 & 9 & \\
\hline Race/ethnicity & & & & & & & & \\
\hline Non-Hispanic White & 3,665 & 78 & 1,470 & 81 & 0.003 & 1,191 & 83 & $<0.0001$ \\
\hline African American & 592 & 13 & 184 & 10 & & 122 & 8 & \\
\hline Hispanic & 218 & 5 & 94 & 5 & & 62 & 4 & \\
\hline Asian & 209 & 4 & 78 & 4 & & 65 & 5 & \\
\hline
\end{tabular}

CCI Charlson comorbidity index, CoI column, Dx diagnosis, $T x$ treatment, $T x 1$ first-line treatment, $T x 2$ second-line treatment ${ }^{*} p$ measures the statistical significance of the difference between column percentages of no Tx group and another group (i.e., Tx 1 only and Tx2 and above)

$\dagger^{\dagger} p$ measures the statistical significance of the difference between column percentages of Tx1 only and Tx2 
chemotherapy treatment as compared to African American race.

Figure 2a shows the 5-year survival curves of elderly mCC patients by number of treatment lines. The standard multivariate Cox regression framework categorized patients into groups according to the number of treatment lines received. The survival benefit associated with $\mathrm{Tx} 2$ as compared to Tx1 was measured by the difference between the areas under the curves of Tx1 and Tx2 (Table 2). The mean survival difference between the Tx2 and Tx1 groups was 0.76 years (95\% CI 0.62-0.89).
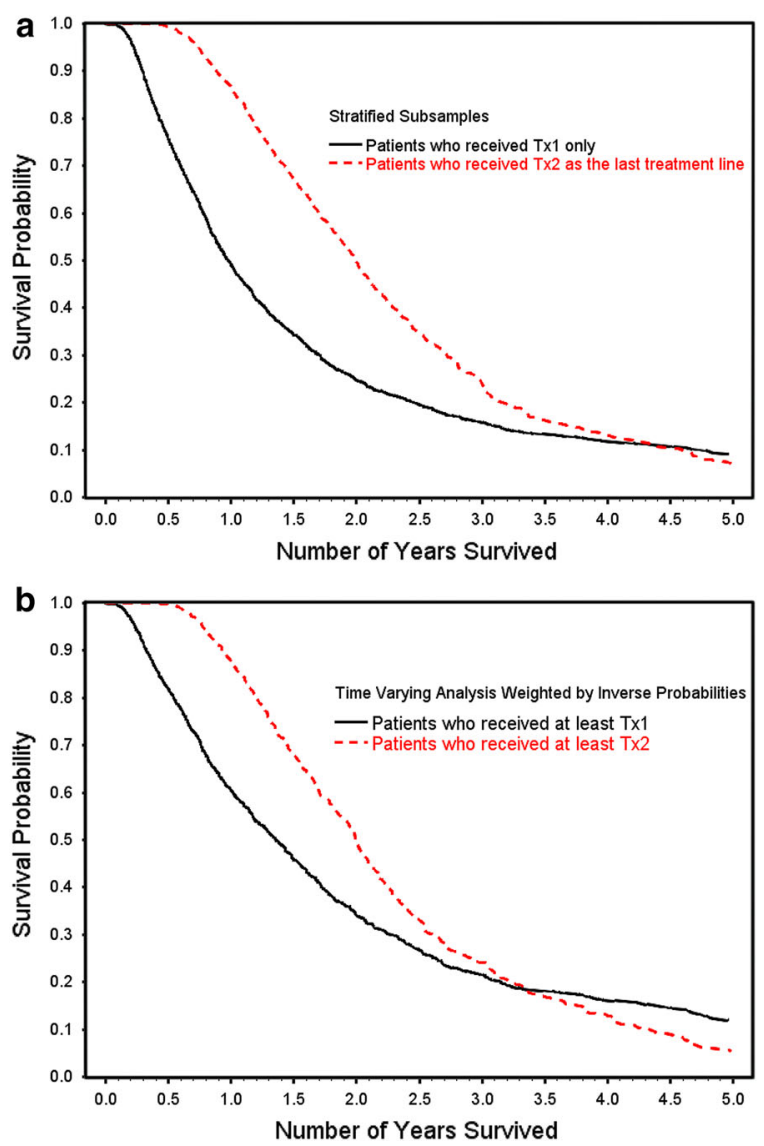

Fig. 2 Cox regression results survival analysis: a stratified analysis; $\mathbf{b}$ time-varying analysis. The black and red lines represent the survival probabilities over time for patients who received $\mathrm{T} x 1$ only and $\mathrm{T} \times 2$ as the last treatment line, respectively. $T x 1$ first-line treatment, $T \times 2$ second-line treatment
Figure $2 \mathrm{~b}$ shows the 5-year survival curves calculated from the time-varying IPW Cox regression framework. The survival benefit associated with $\mathrm{T} x 2$ was measured by calculating the difference between the survival probabilities of patients who were actively receiving Tx 1 and those who were actively receiving $T x 2$. The mean survival benefit obtained from time-varying modeling was 0.33 years (95\% CI $0.19-0.43$ ) (Table 2). Compared with the survival benefit from the static modeling approach, the survival benefit was much smaller in the time-varying model that accounted for the time when patients switched from Tx1 to Tx2.

Table 2 reports the incremental cost associated with Tx2 using the static and the time-varying partitioned IPW cost regression frameworks, respectively. In contrast to the static modeling where patients were grouped according to classifying event (i.e., receipt of Tx2), the time-varying modeling calculated the difference in monthly costs between mCC patients who were actively receiving Tx2 and those who were actively receiving Tx1, adjusting for all patient, provider, and hospital level characteristics. The incremental cost associated with $\mathrm{Tx} 2$ was $\$ 82,615$ (95\% CI $76,883-131,366)$ in the static modeling and $\$ 40,888$ (95\% CI 3,044-44,324) in the timevarying modeling. The reduction in the incremental cost associated with Tx 2 reflected the reduction in the bias introduced by categorizing elderly mCC patients into the Tx2 group while they were in Tx1.

The ICER of Tx2 was $\$ 109,870$ per life year gained (95\% CI 82,513-131,366) in the static modeling and $\$ 123,903$ per life year gained (95\% CI 9,600-216,082) in the time-varying modeling. In contrast to a moderate increase in the ICER from the static modeling to timevarying modeling, there was a substantial increase in the 95\% CIs. The increased 
Table 2 Partitioned IPW: survival/cost analysis of Tx2 treatment (discounting to year 2010 dollars)

\begin{tabular}{|c|c|c|c|c|c|c|}
\hline & \multicolumn{2}{|l|}{$r=0 \%$} & \multicolumn{2}{|l|}{$r=3 \%$} & \multicolumn{2}{|l|}{$r=5 \%$} \\
\hline & Estimate & $95 \%$ CIs & Estimate & 95\% CIs & Estimate & $95 \%$ CIs \\
\hline \multicolumn{7}{|l|}{ Stratified analysis } \\
\hline Incremental cost $(\$)$ & 82,615 & $(76,883-88,827)$ & 91,437 & $(85,108-98,245)$ & 97,780 & $(91,025-104,962)$ \\
\hline $\begin{array}{l}\text { Incremental } \\
\text { survival (years) }\end{array}$ & 0.76 & $(0.62-0.89)$ & 0.76 & $(0.62-0.89)$ & 0.76 & $(0.62-0.89)$ \\
\hline ICER & 109,870 & $(82,513-131,366)$ & 121,614 & $(102,548-145,457)$ & 130,053 & $(109,695-155,534)$ \\
\hline
\end{tabular}

Time-varying analysis

\begin{tabular}{lllllll} 
Incremental cost $(\$)$ & 40,888 & $(3,044-44,324)$ & 44,881 & $(3,205-48,643)$ & 47,734 & $(3,370-51,721)$ \\
$\begin{array}{l}\text { Incremental } \\
\text { survival (years) }\end{array}$ & 0.33 & $(0.19-0.43)$ & 0.33 & $(0.19-0.43)$ & 0.33 & $(0.19-0.43)$ \\
ICER & & & & & & \\
\hline
\end{tabular}

$r(0,3,5 \%)$ represents patients' willingness to postpone costs in the future

$C I$ confidence interval, ICER incremental cost-effectiveness ratio, IPW inverse probability weighting

variability of the ICER is largely due to individual mCC patients' prognoses and the resulting change of treatment status.

Figure 3 shows the impact of timing of initiation of $\mathrm{T} \times 2$ and duration of $\mathrm{T} \times 2$ on the predicted overall incremental cost associated with Tx2. Patients were categorized into three groups according to their timing of initiation of Tx2: early initiation group $(\leq 18$ months since diagnosis), intermediate initiation group (19-36 months), and late initiation group (>36 months). In general, earlier initiation was associated with lower incremental cost for Tx2 among patients who had the same duration of Tx2. The longer the time period that a patient stayed in $\mathrm{Tx} 2$, the higher the cost associated with Tx2.

\section{DISCUSSION}

Our study examined the overall costeffectiveness of second-line chemotherapy among real-world elderly mCC patients. Given the enriched and complex chemotherapy treatment options available to mCC patients, it would have been difficult to recruit and randomize a large number of patients into different treatment strategies using a clinical trial approach. Our retrospective study adopted the IPW method and time-varying Cox regression framework to reduce bias due to patients' selection into treatment. Combined with the time-varying cost analysis, our paper provides an ICER for Tx 2 among elderly mCC patients, which can facilitate the planning and management of chemotherapy and biologics treatments.

A review study summarized the evidence of survival benefit of different regimens in Tx2 from RCTs [24]. A number of conclusions were made regarding the best treatment options after various initial treatments: Tx2 with active regents was superior to best supportive care; following 5-FU failure, IROX was superior to IRI; 


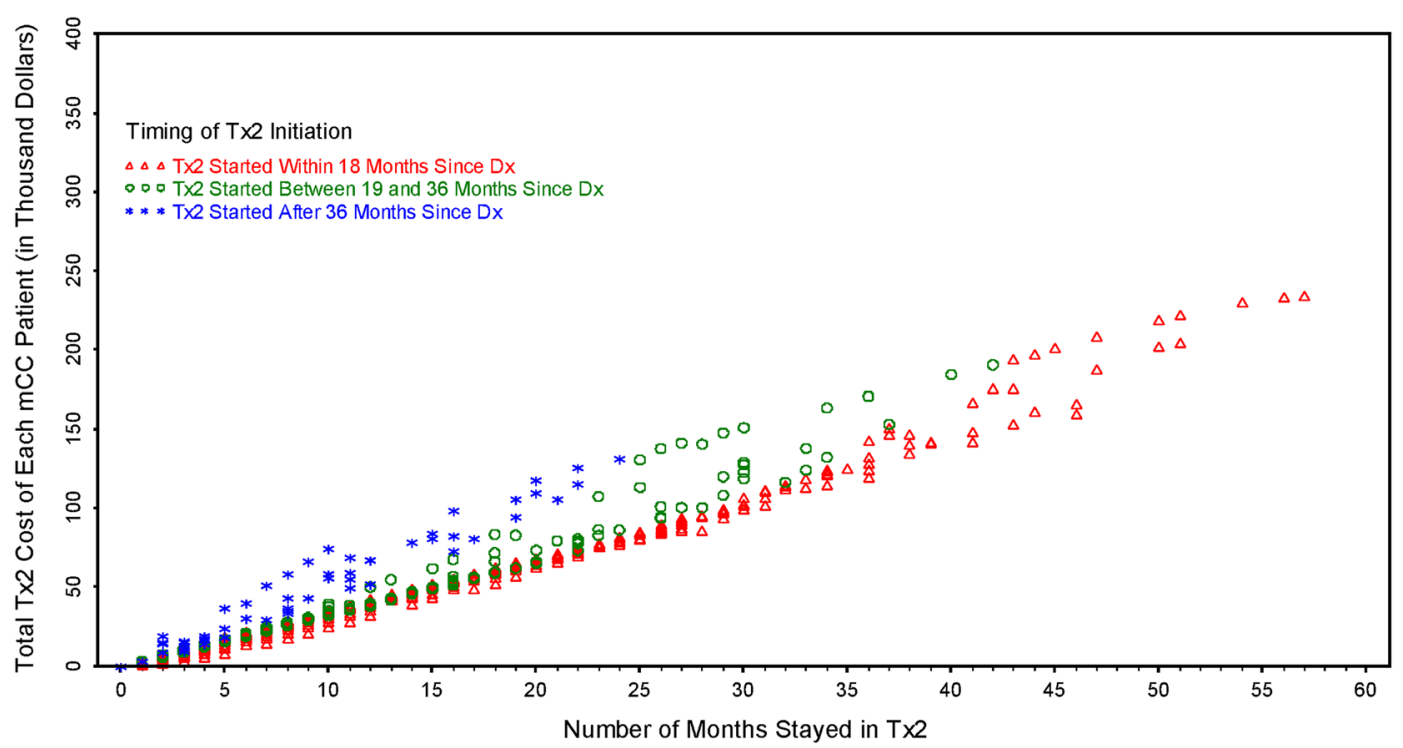

Fig. 3 Individual elderly mCC patients' cost by duration of Tx2. The $x$-axis represents the duration of Tx2 and the $y$-axis represents the incremental cost associated with $\mathrm{T} x 2$.
$D x$ diagnosis, $m C C$ metastatic colon cancer, $T x 1$ first-line treatment, $T \times 2$ second-line treatment

treatment for mCC were not included. Newer biologics that were developed after 2009 and utilized in Tx2 might improve the survival benefit associated with Tx 2 among elderly mCC patients. Depending on the costs associated with these newer regimens in $\mathrm{Tx} 2$, the corresponding ICER might be higher or lower than the estimation from our analysis. In addition, our analysis did not include Medicare prescription drug benefit, due to the availability of the data. The cost analysis used nominal dollars, which did not use the Consumer Price Index to adjust for inflation. We did not discount the survival. Also, utilizing utility weights could also further affect the ICER of Tx2. Moreover, we used a claim-based algorithm to identity chemotherapy treatment lines among Medicare beneficiaries. The survival benefit, costs and cost-effectiveness of Tx2 may not be readily applied to non-elderly populations. Further efforts are needed to examine the cost-effectiveness of $\mathrm{Tx} 2$ among younger populations. 


\section{CONCLUSION}

Less than half of elderly mCC patients initiated chemotherapy/biologics treatment. Among those treated, less than half received secondline treatment. The estimated survival benefit of receiving second-line chemotherapy/biologic treatment is about 4 months, which is consistent with evidence from clinical trials. Time-varying modeling of the survival benefits and associated costs substantially reduced the bias that existed in the traditional stratified analysis. This improved survival was associated with an incremental cost-effectiveness ratio that exceeds the traditional threshold.

\section{ACKNOWLEDGMENTS}

Bayer Healthcare Pharmaceuticals, Inc. provided funding for this project and the article processing charges. No medical writing assistance was received during the preparation of this manuscript. The interpretation and reporting of these data are the sole responsibility of the authors. The authors acknowledge the efforts of the Applied Research Program, NCI; the Office of Research, Development and Information, CMS; Information Management Services (IMS), Inc.; and the Surveillance, Epidemiology, and End Results (SEER) Program tumor registries in the creation of the SEER-Medicare database. Additional contributions: The Pharmaceutical Research Computing center housed in the Pharmaceutical Health Services Research Department at the University of Maryland School of Pharmacy was instrumental for the construction of the analytical data used in the analysis. All named authors meet the ICMJE criteria for authorship for this manuscript, take responsibility for the integrity of the work as a whole, and have given final approval to the version to be published. This study used the linked SEER-Medicare database.

Conflict of interest. C.D. Mullins has research funding sponsored by Bayer and Pfizer, and has a consultant/advisory relationship with Bayer, BMS, Novartis, Jansen/Johnson \& Johnson, and Pfizer. E. Onukwugha has research funding from Bayer and Amgen, and has a consultant/ advisory relationship with Jansen/Johnson \& Johnson. B. Seal is an employee of Bayer and holds stock in Bayer. Zhiyuan Zheng, Nader Hanna, and Kaloyan Bikov have no conflict of interest to declare.

Compliance with ethics guidelines. The analysis in this article is based on previously conducted studies, and does not involve any new studies of human or animal subjects performed by any of the authors. The approved Institutional Review Board protocol number is HP-00049426.

Open Access. This article is distributed under the terms of the Creative Commons Attribution Noncommercial License which permits any noncommercial use, distribution, and reproduction in any medium, provided the original author(s) and the source are credited.

\section{REFERENCES}

1. National Comprehensive Cancer Network (NCCN) Clinical Practice Guidelines in Oncology. Available at: http://www.nccn.org/professionals/physician_ gls/f_guidelines.asp. Accessed Nov 8, 2012.

2. Van Cutsem E, Tejpar S, Vanbeckevoort D, et al. Intrapatient cetuximab dose escalation in metastatic colorectal cancer according to the grade of early skin reactions: the randomized EVEREST study. J Clin Oncol. 2012;30:2861-8. 
3. Peeters M, Price TJ, Cervantes A, et al. Randomized phase III study of panitumumab with fluorouracil, leucovorin, and irinotecan (FOLFIRI) compared with FOLFIRI alone as second-line treatment in patients with metastatic colorectal cancer. J Clin Oncol. 2010;28:4706-13.

4. Fuchs CS, Marshall J, Mitchell E, et al. Randomized, controlled trial of irinotecan plus infusional, bolus, or oral fluoropyrimidines in first-line treatment of metastatic colorectal cancer: results from the BICCC Study. J Clin Oncol. 2007;25:4779-86.

5. Falcone A, Ricci S, Brunetti I, et al. Phase III trial of infusional fluorouracil, leucovorin, oxaliplatin, and irinotecan (FOLFOXIRI) compared with infusional fluorouracil, leucovorin, and irinotecan (FOLFIRI) as first-line treatment for metastatic colorectal cancer: the Gruppo Oncologico Nord Ovest. J Clin Oncol. 2007;25:1670-6.

6. Cassidy J, Clarke S, Díaz-Rubio E, et al. Randomized phase III study of capecitabine plus oxaliplatin compared with fluorouracil/folinic acid plus oxaliplatin as first-line therapy for metastatic colorectal cancer. J Clin Oncol. 2008;26:2006-12.

7. Saltz LB, Clarke S, Díaz-Rubio E, et al. Bevacizumab in combination with oxaliplatin-based chemotherapy as first-line therapy in metastatic colorectal cancer: a randomized phase III study. J Clin Oncol. 2008;26:2013-9.

8. Douillard JY, Siena S, Cassidy J, et al. Randomized, phase III trial of panitumumab with infusional fluorouracil, leucovorin, and oxaliplatin (FOLFOX4) versus FOLFOX4 alone as first-line treatment in patients with previously untreated metastatic colorectal cancer: the PRIME study. J Clin Oncol. 2010;28:4697-705.

9. Van Cutsem E, Peeters M, Siena S, et al. Open-label phase III trial of panitumumab plus best supportive care compared with best supportive care alone in patients with chemotherapy-refractory metastatic colorectal cancer. J Clin Oncol. 2007;25:1658-64.

10. Fuchs CS, Moore MR, Harker G, Villa L, Rinaldi D, Hecht JR. Phase III comparison of two irinotecan dosing regimens in second-line therapy of metastatic colorectal cancer. J Clin Oncol. 2003;21:807-14.

11. Jäger E, Heike $M$, Bernhard $H$, et al. Weekly highdose leucovorin versus low-dose leucovorin combined with fluorouracil in advanced colorectal cancer: results of a randomized multicenter trial. Study Group for Palliative Treatment of Metastatic Colorectal Cancer Study Protocol 1. J Clin Oncol. 1996;14:2274-9.
12. Köhne C-H, Grothey A, Bokemeyer C, Bontke N, Aapro M. Chemotherapy in elderly patients with colorectal cancer. Ann Oncol. 2001;12(4):435-42.

13. Zheng Z, Hanna N, Onukwugha E, Reese ES, Seal B, Mullins CD. Does the type of first-line regimens influence the receipt of second-line chemotherapy treatment? An analysis of 3211 metastatic colon cancer patients. Cancer Med. 2014;3(1):124-33.

14. Bikov KA, Mullins CD, Seal B, Onukwugha E, Hanna N. Algorithm for identifying chemotherapy/biological regimens for metastatic colon cancer in SEER-Medicare. Med Care. 2013 [Epub ahead of print].

15. Charlson ME, Pompei P, Ales KL, MacKenzie CR. A new method of classifying prognostic comorbidity in longitudinal studies: development and validation. J Chronic Dis. 1987;40:373-83.

16. Deyo RA, Cherkin DC, Ciol MA. Adapting a clinical comorbidity index for use with ICD-9-CM administrative databases. J Clin Epidemiol. 1992;45:613-9.

17. Davidoff AJ, Tang M, Seal B, Edelman MJ. Chemotherapy and survival benefit in elderly patients with advanced non-small-cell lung cancer. J Clin Oncol. 2010;28:2191-7.

18. Suissa S. Immortal time bias in pharmacoepidemiology. Am J Epidemiol. 2008;167:492-9.

19. Davidoff AJ, Rapp T, Onukwugha E, et al. Trends in disparities in receipt of adjuvant therapy for elderly stage III colon cancer patients: the role of the medical oncologist evaluation. Med Care. 2009;47:1229-36.

20. Lin DY. Linear regression analysis of censored medical costs. Biostatistics. 2000;1:35-47.

21. Young TA. Estimating mean total costs in the presence of censoring: a comparative assessment of methods. Pharmacoeconomics. 2005;23:1229-42.

22. Griffiths RI, Gleeson ML, Danese MD, O'Hagan A. Inverse probability weighted least squares regression in the analysis of time-censored cost data: an evaluation of the approach using SEERMedicare. Value Health. 2012;15:656-63.

23. Lipscomb J, Drummond $M$, Fryback D, Gold $M$, Revicki D. Retaining, and enhancing, the QALY. Value Health. 2009;12(Suppl. 1):S18-26.

24. Guglielmi AP, Sobrero AF. Second-line therapy for advanced colorectal cancer. Gastrointest Cancer Res. 2007;1:57-63. 